\title{
Molecular-dynamic investigation of the influence of initial temperature on the character of shock-wave processes in silicon carbide nanocluster
}

\author{
Andrey Utkin ${ }^{1, *}$, Vasily Fomin ${ }^{1}$, and Igor Golovnev ${ }^{1}$ \\ ${ }^{1}$ Khristianovich Institute of Theoretical and Applied Mechanics SB RAS, Novosibirsk, 630090, \\ Russia
}

\begin{abstract}
In the present study, using the molecular dynamics method, we investigated the impact interaction of a spherical cluster of $3 \mathrm{C}-\mathrm{SiC}$ silicon carbide with a rigid wall at a wide range of velocities. The influence of cluster initial temperature on the fracture process was analyzed.
\end{abstract}

\section{Introduction}

Silicon carbide is a promising and interesting material due to its excellent chemical and physical stability, low density, high strength, and high thermal conductivity. It is widely used in the manufacture of various friction mechanisms and optoelectronic devices, gas turbines and armor protection materials. Silicon carbide is also used in electronic devices designed for use in adverse environmental conditions.

However, many issues related to the production and processing of silicon carbide have not been well studied. For example, in the case of single-point diamond turning, hightemperature microscale phenomena which occur between the tool and the sample being processed assume a crucial significance [1].

One of the features of silicon carbide is the presence of a huge number of polymorphic modifications [2]. Under the influence of intense external loads various phase transformations can occur in silicon carbide [3]. These phenomena should be taken into account in the numerical simulations of practical problems.

Experimental studies of these processes are complicated by the scale of phenomena, both in time and in space $[2,4]$. Thus, it is urgent to conduct numerical experiments, in particular, molecular dynamics modelling $[5,6]$.

The aim of this work was to study the influence of initial temperature of a spherical ceramic cluster on the character of subsequent physical processes during high-velocity impact with a rigid wall, at a wide range of velocities.

* Corresponding author: utkin@itam.nsc.ru 


\section{Methodology and Physical System}

As a physical system, we considered a spherical cluster of silicon carbide with a zinc blende structure (3C-SiC) that impacted a rigid wall at different velocities. For numerical modelling, we chose cluster with diameter $130 \AA$ (the number of atoms was 113023). Spherical cluster was cut out from a perfect cubic sample of silicon carbide, while the diagonal of this cube, passing through the origin, coincided with the crystallographic direction $[1,1,1]$. Then, using the artificial viscosity method, we found the coordinates and atomic momenta corresponding to the minimum potential energy of the structure. The system was heated using a stochastic method. This method, unlike others in which random forces act on atoms, allowed one to simulate the absorption of energy quanta with random momenta by atoms (analogue of thermal radiation absorption) [7]. In the presented study, the cluster was heated to the following temperatures: $176 \mathrm{~K}, 345 \mathrm{~K}, 688 \mathrm{~K}$ and $1008 \mathrm{~K}$.

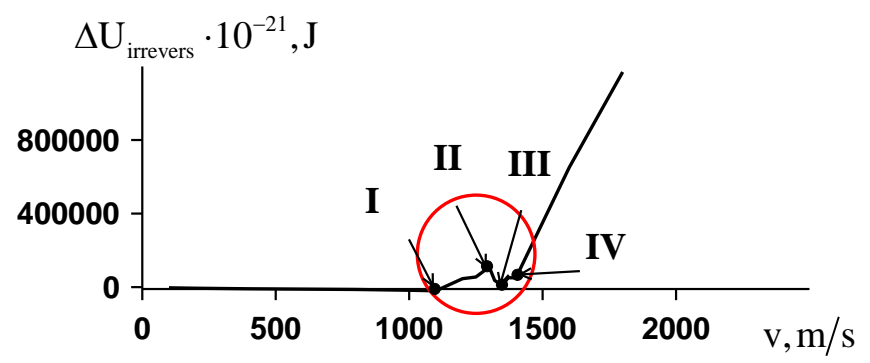

Fig. 1. Irreversible increment of potential energy versus the impact velocity for initial temperature $\mathrm{T}=0 \mathrm{~K}$.

An absolutely rigid wall was modeled with the repulsive branch of the Lennard-Jones potential:

$$
W_{i}=4 e_{L J}\left(d_{L J} /\left(x_{i}-x_{\min }\right)\right)^{8}
$$

where the potential parameters are $e_{L J}=3 \cdot 10^{-21} \mathrm{~J}$ and $d_{L J}=1 \dot{A}$, and $x_{i}$ is the atom position along the $\mathrm{X}$ axis. The wall is located in the plane $x_{\min }=0$, the cluster impacts with the wall from the side of positive values of $\mathrm{X}$ and its initial velocity is negative.

Equations of motion were integrated using the second-order Velocity Verlet scheme [8]. The time step in the numerical calculation was $10^{-16} \mathrm{sec}$. For all numerical simulations parallel molecular dynamics algorithms and codes based on graphics processing units (GPU) were developed and implemented [9-11].

The main advantage of the molecular dynamics method (MDM) for modelling rapid structural and phase transformations at the microscale is that the MDM does not require any assumptions of the nature of the processes being studied, and the thermodynamic properties of the material are determined by the interatomic interaction potential $[4,12]$.

In the present work, we used the potential proposed by P. Vashishta, R. K. Kalia and A. Nakano [13-16]. This potential is based on the Stillinger-Weber formalism (the sum of twobody and three-body terms), and is well-tested for modelling various shock-wave phenomena in ceramic materials. The potential correctly describes the fracture of various polymorphic forms of silicon carbide, and the waves of structural transformations in the substance, under the influence of external loading.

To analyze the collision dynamics, the following macro parameters of the system were calculated: the velocity $V_{c}$ and the energy $E_{c}$ of the centre of mass of the crystal, the force 
$F_{w}$ of the interaction with a wall, the potential component of the internal energy $\Delta U_{\text {in }}=U-U_{0}$, and the kinetic component of the internal energy $E_{K \text { in }}$.
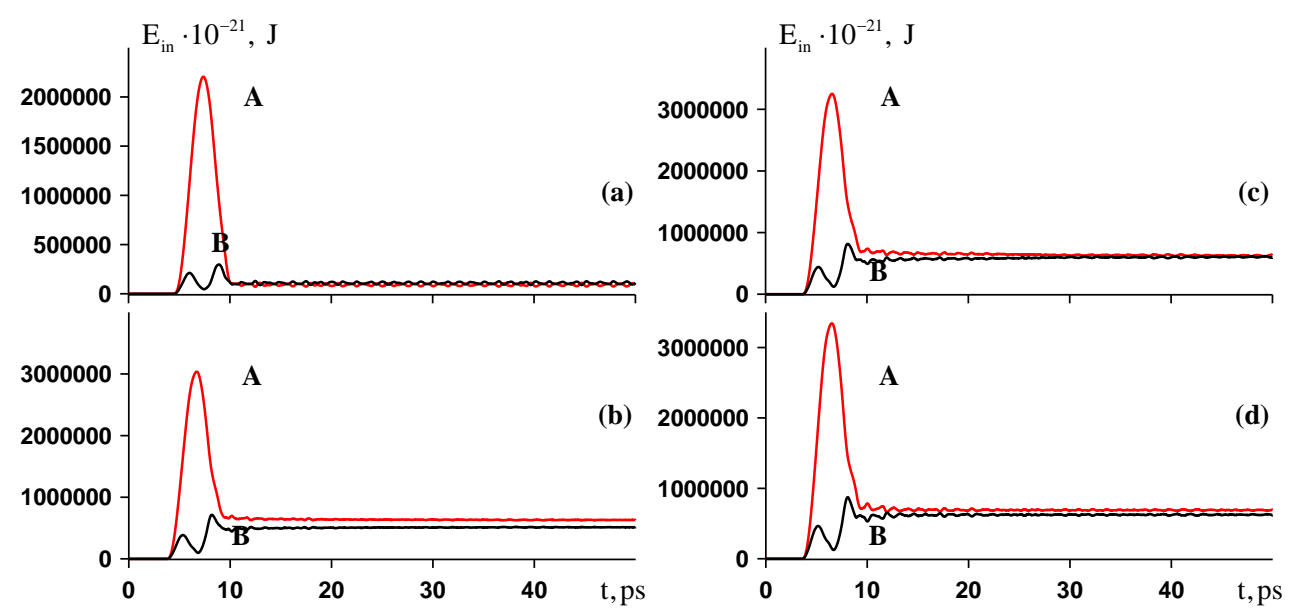

Fig. 2. The internal potential energy (A) and the kinetic energy of the chaotic motion of atoms (B) versus time. Initial impact velocity: (a) $-1100 \mathrm{~m} / \mathrm{s}$, (b) $-1300 \mathrm{~m} / \mathrm{s}$, (c) $-1350 \mathrm{~m} / \mathrm{s}$, (d) - $1370 \mathrm{~m} / \mathrm{s}$.

The atomic structure of the material was analyzed using Adaptive Common Neighbour Analysis (a-CNA) [17,18]. The visualization software OVITO was used to visualize the simulation results [19].

\section{Results}

A behavioural analysis of the energy parameters dependent on the initial impact velocity made it possible to determine the region of irreversible deformations. The irreversible increment in the potential energy (or residual potential energy) of the cluster is a very informative characteristic.

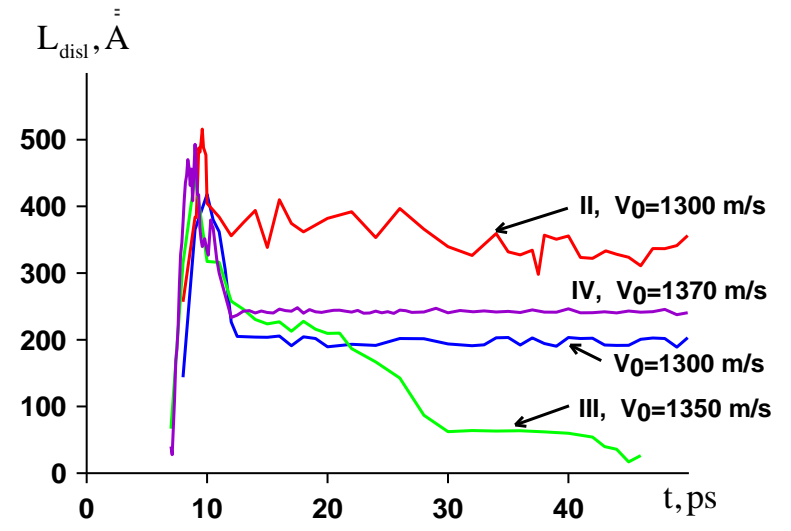

Fig. 3. Total length of dislocations in the cluster versus time.

The system under investigation, in its final state after impact with a rigid wall, is in a local extremum of the potential energy $U_{\text {final }}$ that differs from the initial value of potential energy in a perfect crystal $U_{0}$ at a temperature $\mathrm{T}=0, \mathrm{~K}$. Thus, the irreversible increment of potential energy is defined as: 


$$
\Delta U_{\text {irrevers }}=\left.U_{\text {final }}\right|_{T=0 K}-\left.U_{0}\right|_{T=0 K}
$$

To calculate the final energy $\left.U_{\text {final }}\right|_{T=0 K}$, an additional cooling of the cluster after its impact interaction with the wall was carried out using the artificial viscosity method [20]. Figure 1 shows the irreversible increment of potential energy of the cluster as a function of the impact velocity at initial temperature $\mathrm{T}=0, \mathrm{~K}$.

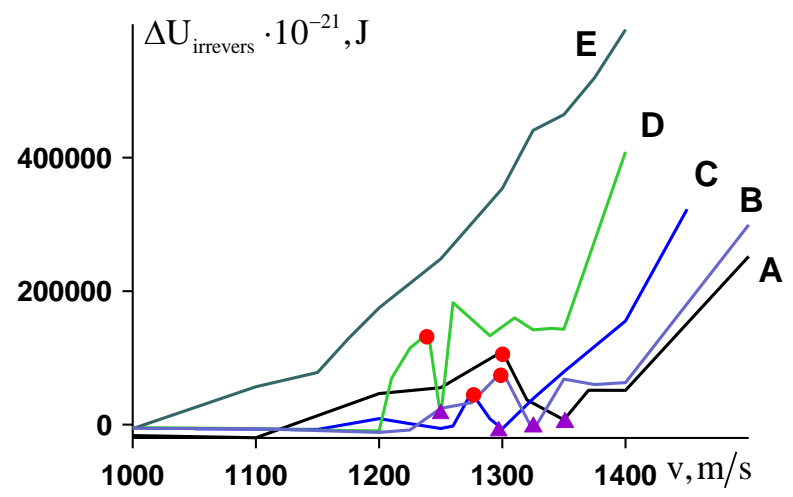

Fig. 4. Irreversible increment of potential energy versus the impact velocity for different cluster initial temperatures: A - 0 K, B - $176 \mathrm{~K}, \mathrm{C}-345 \mathrm{~K}, \mathrm{D}-688 \mathrm{~K}, \mathrm{E}-1008 \mathrm{~K}$.

It can be seen that the impact led to the appearance of minor, but still irreversible, deformations, even in the low impact velocities area. We established a non-monotonic increase $\Delta U_{\text {irrevers }}$ - a characteristic peak appeared at velocities of more than $1000 \mathrm{~m} / \mathrm{s}$; (this area is indicated by a circle in all figures). To clarify the mechanism of such anomalous behaviour, it is necessary to analyze the time dependences of the energy characteristics at feature points that limited the peaks. Such components of the internal energy as the potential component of the internal energy and the kinetic energy of the chaotic motion of atoms will be the most informative ones. For the initial velocity of $1100 \mathrm{~m} / \mathrm{s}$ (point I, Fig. 1), the oscillation of $\Delta U_{\text {in }}$ and $E_{K \text { in }}$ about a certain value identical for these characteristics was observed after collision (about 10 ps) (Fig. 2a). This pattern of wave processes in a cluster is typical for a state that is close to thermodynamic equilibrium. Therein, the temperature fluctuates around $50 \mathrm{~K}$.

A completely different picture can be observed at the local maximum of the peak at an initial velocity of $1300 \mathrm{~m} / \mathrm{s}$ (point II, Fig. 1). After collision (about 9-10 ps), the values of $\Delta U_{\text {in }}$ and $E_{K \text { in }}$ also oscillated with approximately the same amplitude, but about a different mean value - and this value was much higher for $\Delta U_{\text {in }}$ (Fig. 2b). This suggests that the system was trapped in a quasi-stable minimum of the potential energy that lies above the ground state. Therein, the temperature fluctuated about a low value of $220 \mathrm{~K}$.

A non-typical development of the process occurred at a velocity of $1350 \mathrm{~m} / \mathrm{s}$, corresponding to a local minimum at point III in Fig. 1. In the interval up to $30 \mathrm{ps}$, there was a uniform decrease in the potential energy and an increase in the average kinetic energy of the chaotic motion of atoms in accordance with the law of conservation of energy (Fig. 2c). This, in its turn, was confirmed by an increase in system temperature. After $30 \mathrm{ps,} \mathrm{the}$ mean values of the energy characteristics coincided and fluctuated about the same mean value. With increasing impact velocity, the irreversible increment in the potential energy began to increase again. As an example, Fig. 2d shows the dependencies for the velocity of $1370 \mathrm{~m} / \mathrm{s}$ (point IV in Fig. 1). 
Similar to the impact velocity of $1300 \mathrm{~m} / \mathrm{s}$, the values of $\Delta U_{\text {in }}$ and $E_{K \text { in }}$ after collision oscillated with approximately the same amplitude, but about a different mean value.

The use of dislocation analysis made it possible to analyze the state of matter after impact at different time points for different initial velocities [21]. It was found that the state of matter corresponding to point III (Fig. 1) was characterized by a rapid decrease in the number and total length of dislocations in the cluster over time (Fig. 3). As the total length of dislocations decreased, the energy characteristics began to equalize (see Fig. 2c). A similar study of the state of matter at points II and IV showed that the total length of dislocations formed in the cluster after the impact varied in time insignificantly.

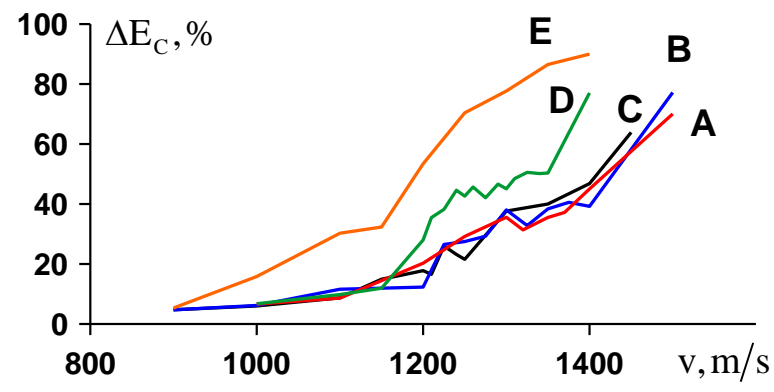

Fig. 5. Energy dissipation coefficient versus the impact velocity for different cluster initial temperatures: A - $0 \mathrm{~K}, \mathrm{~B}-176 \mathrm{~K}, \mathrm{C}-345 \mathrm{~K}, \mathrm{D}-688 \mathrm{~K}, \mathrm{E}-1008 \mathrm{~K}$.

By analogy with numerical simulation at an initial zero temperature, a set of numerical experiments on the impact of a heated cluster was performed. Figure 4 shows irreversible increment of the potential energy as a function of the impact velocity for different initial temperatures. We established a non-monotonic increase of $\Delta U_{\text {irrevers }}$ for $\mathrm{T}=176 \mathrm{~K}$, $\mathrm{T}=345 \mathrm{~K}$, and $\mathrm{T}=688 \mathrm{~K}$. As well as for zero initial temperature characteristic peak appeared at velocities of more than $1000 \mathrm{~m} / \mathrm{s}$; (local maximum of this peak is indicated by a circle and local minimum is indicated by triangle). It followed from presented dependencies that increasing cluster initial temperature resulted in peaking at lower initial velocity values. A further increasing of the initial cluster temperature led to the disappearance of the peak in the dependence of irreversible increment of potential energy from the impact velocity $(\mathrm{T}=1007 \mathrm{~K})$. It can be seen that the impact led to the appearance of noticeable and irreversible deformations, even in the low impact velocities area. Thus, for impact velocity $1300 \mathrm{~m} / \mathrm{s}$, increasing of initial temperature from $176 \mathrm{~K}$ to $1007 \mathrm{~K}$ led to increasing of irreversible increment of potential energy more than 10 times. The use of dislocation analysis made it possible to analyze the state of matter after impact at different time points for velocities corresponded to the local maximum and minimum of $\Delta U_{\text {irrevers }}$ peaks.

It was found that the state of matter corresponding to the local minimum points (triangles from Figure 4) was characterized by a rapid decrease in the number and total length of dislocations in the cluster over time (as for zero initial temperature). As the total length of dislocations decreased, the internal potential energy and the kinetic energy of the chaotic motion of atoms began to equalize.

It is easy to see (Fig. 5) that a further increase in initial temperature for the same impact velocities was accompanied by a sharp increase in the energy dissipation coefficient that is defined as

$$
\Delta E_{c}=\left(\left(E_{c 0}-E_{c f i n a l}\right) / E_{c 0}\right) \cdot 100 \%
$$


where $E_{c 0}$ and $E_{c f i n a l}$ are the kinetic energy of the cluster centre of mass before and after collision, respectively.

From the presented dependences it follows that an increase in initial temperature led to the deterioration in structural behaviour of the samples.

\section{Conclusion}

Within the MDM framework, we carried out a numerical simulation of the impact of a spherical cluster of silicon carbide with an absolutely rigid wall. The influence of cluster initial temperature on the fracture pattern has been studied.

A non-monotonic increase in the residual potential energy with increasing impact velocity has been found. An increase in the initial temperature of the cluster resulted in peaking at lower initial velocity values. A further increasing of the initial cluster temperature led to the disappearance of the peak. An increase in the initial temperature was accompanied by an increase in the energy dissipation coefficient for the same values of the initial velocity and the appearance of irreversible deformation at lower impact velocities.

The present investigation was supported by Presidium RAS, program 27 Fundamental aspects of supercomputing for simulating complex practical problems and by RFBR grant \# 18-41-540013 r_a. The Siberian Branch of the Russian Academy of Sciences (SB RAS) Siberian Supercomputer Center is gratefully acknowledged for providing supercomputer facilities. This research was supported in through computational resources provided by the Shared Facility Center "Data Center of FEB RAS" (Khabarovsk).

\section{References}

1. S. Goel, J. Phys. D: Appl. Phys. 47, 243001 (2014)

2. J. Fan, P.K. Chu, Silicon Carbide Nanostructures Fabrication, Structure, and Properties (Springer International Publishing, Berlin, 2014)

3. M. Yoshida, A. Onodera, M. Ueno, K. Takemura, O. Shimomura, Phys. Rev. B. 48, 10587-10590 (1993)

4. S.G. Psakhie, K.P. Zolnikov, D.S. Kryzhevich, A.V. Korchuganov, Sci. Rep. 9, 3867 (2019)

5. A.E. Buzyurkin, E.I. Kraus, Y.L. Lukyanov, Thermal Science 23, 471(2019)

6. A.E. Buzyurkin, E.I. Kraus, Ya.L. Lukyanov, AIP Conference Proceedings 1770, 030091 (2016)

7. I. F. Golovnev, E. I. Golovneva, V. M. Fomin, Phys. Mesomech. 11, 19 (2008)

8. D. Frenkel, B. Smit, Understanding Molecular Simulation: From Algorithms to Applications (Elsevier Academic Press, Amsterdam, 2001)

9. M.S. Ozhgibesov, T.S. Leu, C.H. Cheng, A.V. Utkin, Journal of Molecular Graphics and Modelling 45, 45 (2013)

10. M.S. Ozhgibesov, A.V. Utkin, V.M. Fomin, T.S. Leu, C.H. Cheng, Int. J. Comp. Mat. Sci. Eng. 01, 1250007 (2012)

11. A.V. Utkin, Mathematica Montisnigri 39, 101 (2017)

12. A.V. Utkin, V.M. Fomin, AIP Conference Proceedings. 1893 (1), 030018 (2017)

13. P. Vashishta, R.K. Kalia, A. Nakano, J.P. Rino, J Applied Physics 101, 103515 (2007)

14. M. Mishra, I. Szlufarska, Acta Materialia 57, 6156 (2009) 
15. J. Zhang, P.S. Branicio, Procedia Engineering 75, 150 (2014)

16. F. Shimojo, I. Ebbsjö, R.K. Kalia, A. Nakano, J.P. Rino, P. Vashishta, Physical Review Letters 84, 3338 (2000)

17. A. Stukowski, Modell. Simul. Mater. Sci. Eng. 20, 045021 (2012)

18. J.D. Honeycutt, H.C. Andersen, J. Phys. Chem. 91 (19), 4950 (1987)

19. A. Stukowski, Modell. Simul. Mater. Sci. Eng. 18, 015012 (2010)

20. I.F. Golovnev, E.I. Golovneva, V.M. Fomin, Phys. Mesomech. 6, 41 (2003).

21. A. Stukowski, V.V. Bulatov and A. Arsenlis, Modelling Simul. Mater. Sci. Eng. 20, 085007 (2012) 